\title{
Energy Release During Slow Long-Duration Flares Observed by RHESSI
}

\author{
U. Ba̧k-Stȩślicka · T. Mrozek • S. Kołomański
}

Received: 1 December 2010 / Accepted: 23 May 2011 / Published online: 6 July 2011

(C) The Author(s) 2011. This article is published with open access at Springerlink.com

\begin{abstract}
Slow long-duration events (SLDEs) are flares characterized by the long duration of their rising phase. In many such cases the impulsive phase is weak without typical shortlasting pulses. Instead, smooth, long-lasting hard X-ray (HXR) emission is observed. We analyzed hard X-ray emission and morphology of six selected SLDEs. In our analysis we utilized data from the RHESSI and GOES satellites. The physical parameters of HXR sources were obtained from imaging spectroscopy and were used for the energy balance analysis. The characteristic decay time of the heating rate, after reaching its maximum value, is very long, which explains the long rising phase of these flares.
\end{abstract}

Keywords Sun: corona $\cdot$ Flares $\cdot$ X-rays

\section{Introduction}

A long-duration event (LDE) is a flare characterized by slow decrease of soft X-ray (SXR) emission. SXR images of such flares were observed since Skylab and Solar Maximum Mission (SMM) observations (Sheeley et al., 1975). Those observations indicated that LDEs usually occurred in high arcade of loops (Kahler, 1977). Yohkoh observed flares with temporal and spatial resolution better than Skylab and SMM, so that LDEs became the object of study by many authors (Tsuneta et al., 1992; Feldman et al., 1995; Hudson, Acton, and Freeland, 1996; Tomczak, 1997; Harra-Murnion, Schmieder, and van Driel-Gesztelyi, 1998; Isobe et al., 2002; Phillips, Feldman, and Harra, 2005; Kołomański, 2007a, 2007b).

U. Ba̧k-Stȩślicka (凶) · T. Mrozek · S. Kołomański

Astronomical Institute, University of Wrocław, ul. Kopernika 11, 51-622 Wrocław, Poland e-mail: bak@astro.uni.wroc.pl

T. Mrozek

e-mail: mrozek@astro.uni.wroc.pl

S. Kołomański

e-mail: kolomanski@astro.uni.wroc.pl 
Among LDEs are flares with their rise phase lasting longer than in others, i.e. more than $30 \mathrm{~min}$. Such flares are called slow long-duration events (SLDEs) and their characteristic feature is that their impulsive phase is weak or does not exist (Hudson and McKenzie, 2000, 2001). In many cases long-lasting hard X-ray (HXR) emission was observed and its non-thermal character was confirmed (Hudson and McKenzie, 2000). During the Yohkoh mission (1991-2001), Geostationary Operational Environmental Satellites (GOES) registered more than 250 SLDEs (Ba̧k-Stȩślicka, 2007). The well-known examples of slow LDE are 21 February 1992 (Tsuneta et al., 1992) and 20 January 1999 events in which supra-arcade downflows were discovered (McKenzie and Hudson, 1999; McKenzie, 2000).

GOES observations were useful for finding SLDEs. Ba̧k-Stęślicka and Jakimiec (2005) have shown that the time interval, $\Delta t$, between the temperature and emission measure maxima can be used as a measure of the duration of the rising phase. This method is independent of the background level and gives us a more reliable value of the duration of the rising phase.

Bạk-Stęślicka (2007) used the data from the Yohkoh Hard X-ray Telescope (HXT, Kosugi et al., 1991) and analyzed several limb or near-the-limb slow LDEs. In most of the cases long-lasting HXR emission without typical, short lasting pulses was observed. The HXR emission sources in low energy channels were spatially correlated with the tops of loops seen in SXR images. In a few cases high energy emission sources were observed near the footpoints of loops.

Ba̧k-Stęślicka and Jakimiec (2005) used the images taken with the Yohkoh Soft X-ray Telescope (SXT, Tsuneta et al., 1991) and analyzed the morphology of loop-top sources (LTS) and calculated their physical parameters. This analysis indicated that most of the slow LDEs occurred in high or mid-high structures $(h \approx 20-50 \mathrm{Mm})$. The loop-top sources were characterized by low temperature $(T<10 \mathrm{MK})$, low density $\left(N \approx 10^{10} \mathrm{~cm}^{-3}\right)$ and large size $\left(r>7 \times 10^{8} \mathrm{~cm}\right)$. The thermal energy release rate was small, below $1 \mathrm{erg} \mathrm{cm}^{-3} \mathrm{~s}^{-1}$ and decreased very slowly with time after reaching its maximum value.

Slow LDEs could not be investigated in more detail because of limited sensitivity of SXT and low spectral resolution of HXT. To overcome these instrumental limitations we decided to use Reuven Ramaty High Energy Solar Spectroscopic Imager (RHESSI) observations. RHESSI allows us to investigate spatially resolved HXR emission of SLDEs with $1 \mathrm{keV}$ energy resolution, and to distinguish between thermal and non-thermal natures of LTS and calculate its physical parameters. Here we present the analysis of six selected slow LDEs.

\section{Observations}

Our analysis is based on RHESSI (Lin et al., 2002) and GOES X-ray Sensor (XRS) data, supported by Solar and Heliospheric Observatory Extreme UV Imaging Telescope (SOHO/EIT, Delaboudinière et al., 1995), Transition Region and Coronal Explorer (TRACE, Handy, Acton, and Kankelborg, 1999) and GOES Solar X-Ray Imager (SXI, Hill et al., 2005) observations. RHESSI is a rotating Fourier imager with nine detectors made of pure germanium crystals (Lin et al., 2002). The detectors record the energy and time of arrival of each HXR photon detected. Pairs of grids placed ahead of the detectors and the rotation of the whole satellite (4 s period) cause modulation of HXR radiation coming from solar sources. It is possible to reconstruct HXR images (Hurford, Schmahl, and Schwartz, 2002) using several available algorithms.

We analyzed the rise phase of SLDE events using images reconstructed with the PIXON algorithm (Puetter and Yahil, 1999 and references within). The images were reconstructed 
in time intervals of 20-40 s covering the whole rise phase. Energy intervals were narrow, usually $1-2 \mathrm{keV}$. Such intervals were chosen since we were interested in the imaging spectroscopy of HXR sources. The imaging spectroscopy we performed has an advantage in comparison to "standard" spectroscopy based on the fluxes measured over the whole Sun. Namely, for the rotating Fourier imager the background photons do not influence the modulation profile, so that the analysis of fluxes can be done more precisely.

\section{Data Analysis}

For our analysis we selected six limb or near-the-limb solar flares with slow rise phases (the rise phases lasted between $25-150 \mathrm{~min}$ ). We calculated the temperature $T_{\mathrm{G}}$, emission measure $\mathrm{EM}_{\mathrm{G}}$ and time difference, $\Delta t$, between their maxima using GOES/XRS data. We obtained the physical parameters of the loop-top sources using RHESSI data.

\subsection{Physical Parameters of LTS}

A loop-top source was defined as an area of HXR emission bounded by the intensity isoline equal to $50 \%$ of the intensity of the brightest pixel. We calculated the source area for a given time interval and different energy ranges. The mean area was used for calculating the mean radius, $r$, assuming a spherical shape of the loop-top source. Using the mean radius (Table 2) we calculated the volume of emitting region for a given time interval. The altitude of the loop-top source was calculated from the centroid of the HXR emission source.

With spatially resolved signals we were able to perform spectroscopy of individual HXR sources (bounded by isocontour equal to $50 \%$ of the intensity of the brightest pixel) using the standard OSPEX package. The best fit to the observed spectra was obtained by assuming a composite of a thermal component, two spectral line complexes (at $6.7 \mathrm{keV}$ and $8.0 \mathrm{keV}$ ), and a single power-law component. Other sets of components, for example a double thermal model instead of the thermal plus power-law model, were also used. In such cases the obtained fits were significantly worse. The values obtained for $T_{R}$ and $\mathrm{EM}_{\mathrm{R}}$ were used to calculate the heating rate during the rise phase of the flares investigated.

Uncertainties in the investigated parameters were calculated as follows. In the case of SLDEs we may assume slow temporal changes of the parameters. Thus, the observed fast and small fluctuations of the parameters observed for consecutive time intervals may be the product of observational errors. After subtracting a slowly varying trend in the size, temperature, and emission measure of the loop-top sources, we calculated the standard deviation for them. We treated the standard deviation as an uncertainty of the parameters. The obtained estimations for uncertainties are about $5 \%$ for the mean radius of the loop-top source, about $15 \%$ for the volume of LTS, less than $5 \%$ for temperature, and less than $20 \%$ for emission measure.

A non-thermal component of the HXR spectrum was observed in four cases. We fitted this component with a double power-law function. We treated a break energy and power law index above this energy as free parameters. The power law index below the break energy was fixed at constant value. The total power in non-thermal electrons above the cut-off energy, $\epsilon_{\mathrm{c}}$, was calculated using a formula given by Aschwanden (2005):

$$
P\left(\epsilon \geq \epsilon_{\mathrm{c}}\right)=4.3 \times 10^{24} \frac{b(\gamma)}{(\gamma-1)} A\left(\epsilon_{\mathrm{c}}\right)^{-(\gamma-1)}\left(\mathrm{erg} \mathrm{s}^{-1}\right) .
$$


Here $b(\gamma) \approx 0.27 \gamma^{3}$ is the auxiliary function calculated in Hudson, Canfield, and Kane (1978), and $A$ is a normalization factor. Since we were interested in a rough estimation of non-thermal power we assumed that the break energy from our fits is close to the cut-off energy.

\subsection{Energy Balance}

We estimated the value of the heating function, $E_{\mathrm{H}}$, for the loop-top sources seen in RHESSI images for all the analyzed flares. In order to calculate the heating rate of LTS we considered its energy balance during the rise phase. Here we consider a time interval, $\Delta t$, after temperature maximum, when the energy release has begun to decrease, but the emission measure is still increasing. Three major cooling processes (expansion, radiation and conduction) where included in this balance. We have

$$
\left(\frac{\mathrm{d} \mathcal{E}}{\mathrm{d} t}\right)_{\mathrm{obs}}=\left(\frac{\mathrm{d} \mathcal{E}}{\mathrm{d} t}\right)_{\mathrm{ad}}-E_{\mathrm{C}}-E_{\mathrm{R}}+E_{\mathrm{H}},
$$

where $\mathcal{E}=3 N k T$ is thermal energy density, $\left(\frac{\mathrm{d} \mathcal{E}}{\mathrm{d} t}\right)_{\text {obs }}$ is the decrease of $\mathcal{E}$ per second estimated from temperature $(T)$ and density $(N)$ values, $\left(\frac{\mathrm{d} \mathcal{E}}{\mathrm{d} t}\right)_{\text {ad }}$ is the decrease due to the adiabatic expansion of plasma in a source, $E_{\mathrm{C}}$ is the energy loss due to thermal conduction, $E_{\mathrm{R}}$ is the radiative loss, and $E_{\mathrm{H}}$ is the heating rate or the rate of thermal energy release. The values of $E_{\mathrm{C}}, E_{\mathrm{R}}$ and $E_{\mathrm{H}}$ are in $\mathrm{erg} \mathrm{cm}^{-3} \mathrm{~s}^{-1}$. Then we calculated:

$-\left(\frac{\mathrm{d} \mathcal{E}}{\mathrm{d} t}\right)_{\mathrm{ad}}=5 k T\left(\frac{\mathrm{d} N}{\mathrm{~d} t}\right)$.

- $E_{\mathrm{C}}=3.9 \times 10^{-7} T^{3.5} /(L r)$, where $r$ is the LTS radius and $L$ is loop semi-length (Jakimiec et al., 1997).

- $E_{\mathrm{R}}=N^{2} \Phi(T)$, where $\Phi(T)$ is the radiative loss function taken from Dere et al. (2009).

We took the height of the LTS above the photosphere, $h$, as an approximation for $L$ in the expression for $E_{\mathrm{C}}$. Since the value of $h$ is always smaller than $L$, the calculated value of $E_{\mathrm{H}}$ is the upper limit.

Uncertainties calculated for these basic parameters allowed us to estimate the errors in $E_{\mathrm{H}}$. In the case of SLDEs $E_{\mathrm{C}}$ is a dominant contributor in the energy balance. It is about an order of magnitude greater than other contributors to $E_{\mathrm{H}}$. The estimated value of the uncertainty in $E_{\mathrm{C}}$ is about $20 \%$. Similar values have been obtained through the analysis of the standard deviation.

For all selected flares we analyzed the time evolution of $E_{\mathrm{H}}(t)$ and calculated the characteristic time $\tau$ of the decrease in $E_{\mathrm{H}}(t)$ after reaching its maximum value:

$$
\frac{1}{\tau}=\frac{\mathrm{d} \ln E_{\mathrm{H}}(t)}{\mathrm{d} t}
$$

\section{Results}

We calculated the temperature, $T_{\mathrm{G}}$, emission measure, $\mathrm{EM}_{\mathrm{G}}$, and the difference between their maxima (Table 1) using GOES/XRS data. For all flares we used RHESSI data to obtain the physical parameters of loop-top sources from spectra fitting. The parameters were used to determine the heating rate and the characteristic time of its decrease after reaching the maximum value. Below we will describe three flares in detail. The results for all flares are given in Table 2. 
Table 1 Data for selected flares.

\begin{tabular}{|c|c|c|c|c|c|c|c|}
\hline \multirow[t]{2}{*}{ Flare } & \multirow[t]{2}{*}{ Date } & \multicolumn{6}{|c|}{ GOES/XRS } \\
\hline & & $\begin{array}{l}\text { Start } \\
{[\mathrm{UT}]}\end{array}$ & $\begin{array}{l}\text { Max } \\
{[\mathrm{UT}]}\end{array}$ & Class & $\begin{array}{l}T_{\mathrm{Gmax}} \\
{[\mathrm{MK}]}\end{array}$ & $\begin{array}{l}\mathrm{EM}_{\mathrm{Gmax}} \\
{\left[10^{48} \mathrm{~cm}^{-3}\right]}\end{array}$ & $\begin{array}{l}\Delta t \\
{[\mathrm{~min}]}\end{array}$ \\
\hline 1 & 24 Oct. 2003 & $02: 19$ & $02: 54$ & M7.6 & 17.8 & 43 & 21 \\
\hline 2 & 18 Nov. 2003 & $09: 23$ & $10: 11$ & M5.4 & 12.8 & 29 & 23 \\
\hline 3 & 13 Jul. 2005 & 14:01 & $14: 49$ & M5.0 & 19.0 & 26 & 39 \\
\hline 4 & 23 Aug. 2005 & $14: 19$ & $14: 44$ & M2.7 & 20.2 & 14 & 20 \\
\hline 5 & 06 Sep. 2005 & $19: 32$ & $22: 02$ & M1.4 & 16.2 & 7.7 & 101 \\
\hline 6 & 25 Jan. 2007 & $06: 33$ & $07: 14$ & C6.3 & 13.0 & 5 & 29 \\
\hline
\end{tabular}

$\Delta t=t\left(\mathrm{EM}_{\mathrm{Gmax}}\right)-t\left(T_{\mathrm{Gmax}}\right)$.
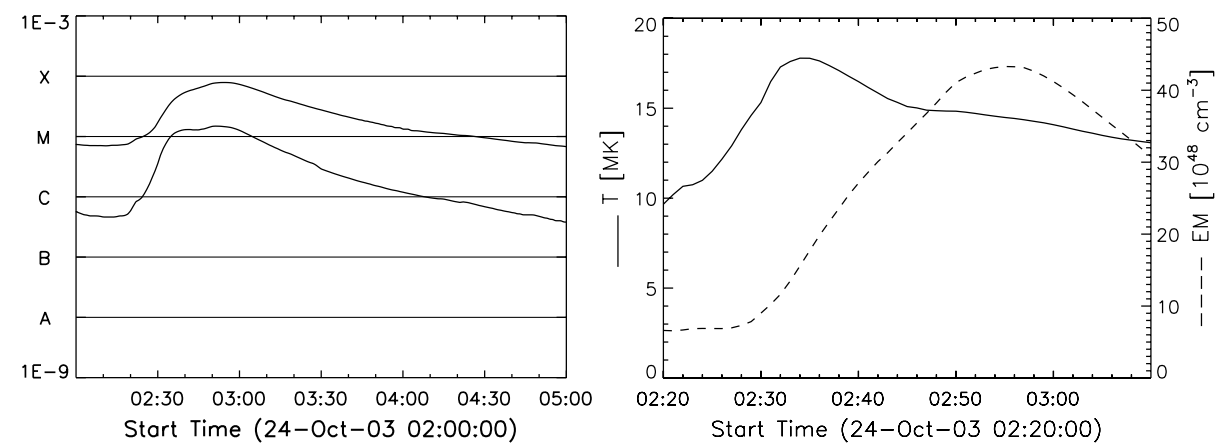

Figure 1 Left: GOES X-ray fluxes (upper curve: $1-8 \AA$, lower curve: $0.5-4 \AA$ ). Right: Temperature and emission measure during the rise phase of the 24 October 2003 flare obtained from the GOES/XRS data.

\subsection{Flare of 24 October 2003}

The flare started at about 02:19 UT in active region NOAA 10486. The SXR flux reached its maximum (M7.6) at 02:54 UT. Two phases are clearly seen (see Figure 1, left panel) in the GOES light curves: the first one is from the beginning up to $\approx 02: 44 \mathrm{UT}$, and the second one after 02:44 UT. We used GOES/XRS data to calculate the temperature $T_{\mathrm{G}}$ and emission measure $\mathrm{EM}_{\mathrm{G}}$ of the whole flare. The temperature increased slowly and reached its maximum value (17.8 MK) at 02:34 UT (Figure 1, right panel). The emission measure reached its maximum value $\left(43 \times 10^{48} \mathrm{~cm}^{-3}\right)$ at $02: 55 \mathrm{UT}$. The difference in time, $\Delta t$, between these two maxima is $21 \mathrm{~min}$.

The flare was well observed by many instruments. A detailed multi-wavelength analysis of this flare was presented by other authors ( $\mathrm{Li}$ and Li, 2008; Joshi et al., 2009). Two phases of this flare are also visible in the RHESSI light curves (Figure 2, left panel).

\subsubsection{First Phase}

RHESSI observed this flare from 02:22 UT. During the first phase the emission up to $25-$ $50 \mathrm{keV}$ range was observed, but there were no short-lasting pulses. The TRACE data helped us to investigate the morphology of the flare. The thermal response function for the TRACE 

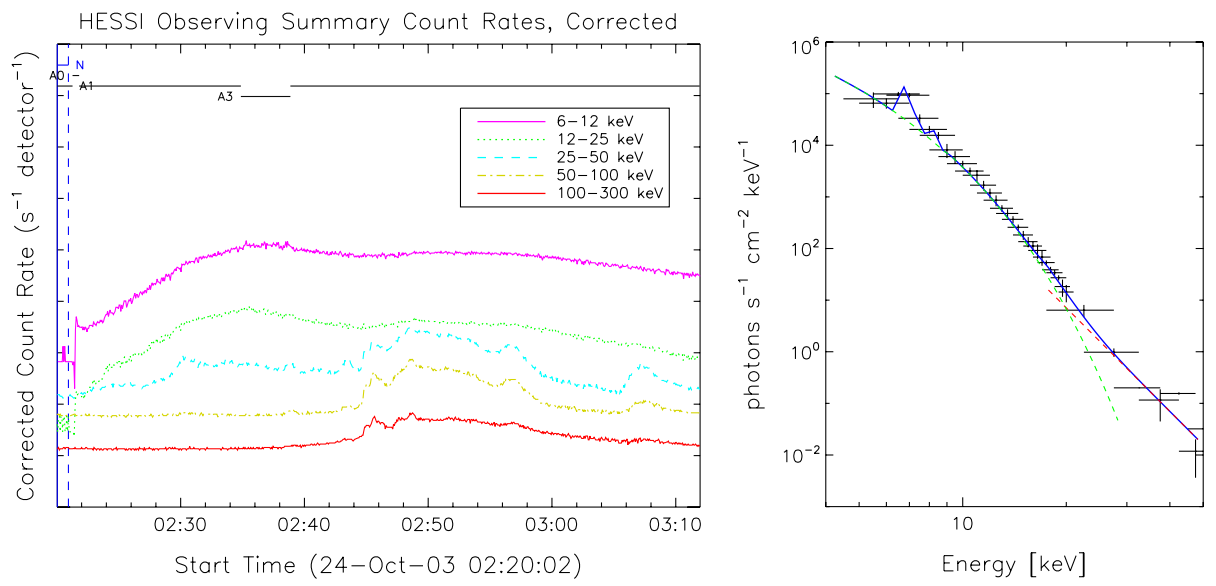

Figure 2 Left:RHESSI light curves of the 24 October 2003 flare. Right: The RHESSI X-ray spectrum of the loop-top source (source N) observed during the 24 October 2003 flare at 02:48 UT (horizontal bars correspond to the energy bin widths). This spectrum was fitted using the thermal component (green curve), two spectral line complexes (at $6.7 \mathrm{keV}$ and $8.0 \mathrm{keV}$ ), and a single power-law component (red curve). The sum of all these models, the best-fit model, is represented by the blue curve.
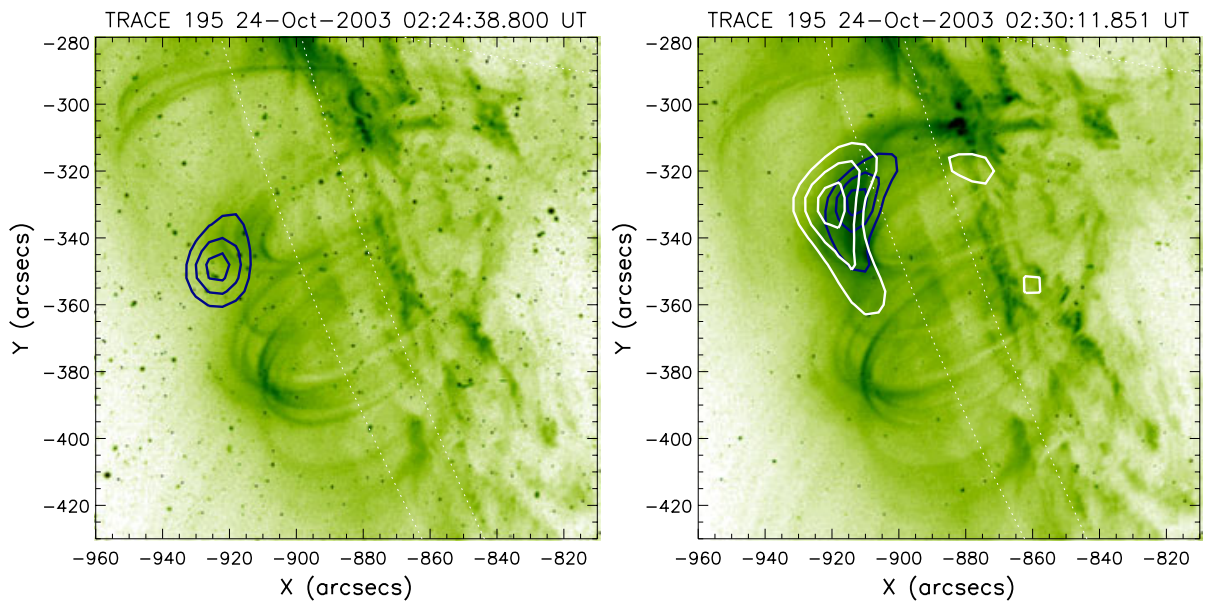

Figure 3 TRACE $195 \AA$ images of the 24 October 2003 flare during the first phase. Contours show the emission in the 10-11 keV range (blue) and 20-25 keV range (white) observed with RHESSI. The contours are for $50 \%, 70 \%$ and $90 \%$ of maximum emission.

$195 \AA$ filter has two distinct peaks: the higher peak for plasma at $T \approx 1-2 \mathrm{MK}$ and the lower peak for significantly hotter plasma $(T \approx 10-30 \mathrm{MK}$, Phillips, Chifor, and Landi, 2005). We carefully co-aligned RHESSI and TRACE $195 \AA$ Amages using SOHO/EIT $195 \AA$ images (Gallagher et al., 2002). The TRACE $195 \AA$ images with RHESSI contours are shown in Figure 3. At the beginning, one source, slightly elongated in the north direction, was observed. A few minutes later, the source (source $\mathrm{N}$ ) was visible at a lower altitude (see Table 2) and was spatially correlated with the very bright loop-top source seen in the TRACE $195 \AA$ images. The hot source N dominated in the emission during the first phase. 

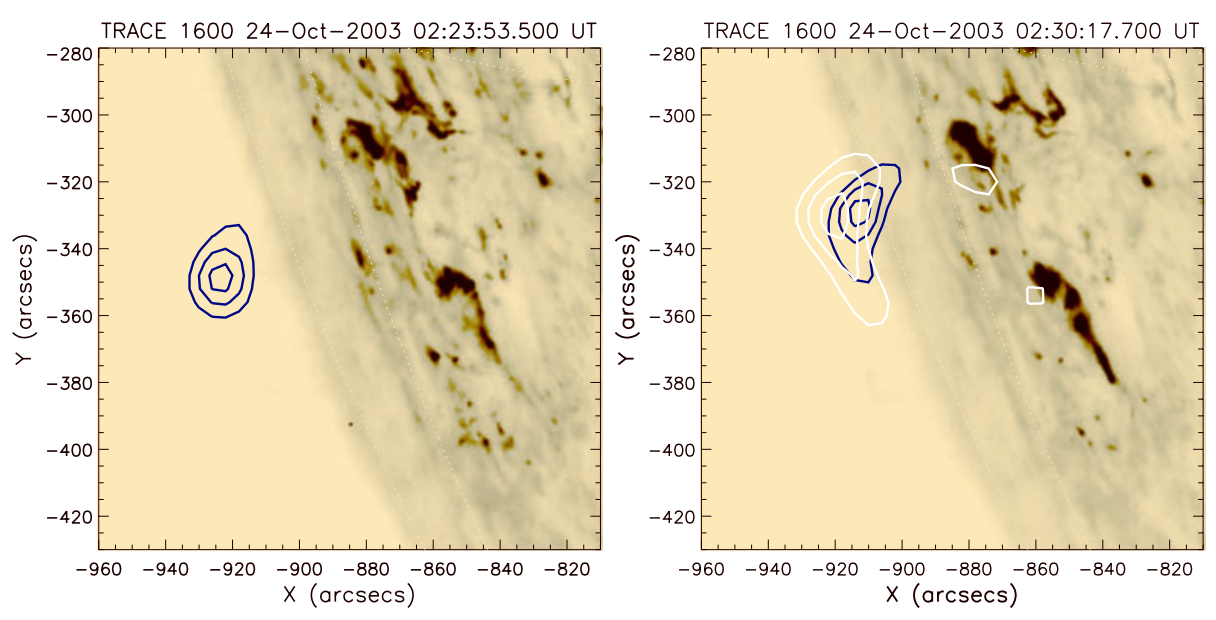

Figure 4 TRACE $1600 \AA$ images of the 24 October 2003 flare during the first phase. Contours show the emission in the 10-11 keV range (blue) and 20-25 keV range (white) observed with RHESSI. The contours are for $50 \%, 70 \%$ and $90 \%$ of maximum emission.

Shrinkage of TRACE and RHESSI loops was previously reported ( $\mathrm{Li}$ and $\mathrm{Li}, 2008$; Joshi et al., 2009). Only at about 02:30 UT the footpoints (FPN in the north direction, FPS in the south direction) were visible and were spatially correlated with the ribbons seen in TRACE $1600 \AA$ A images (Figure 4).

\subsubsection{Second Phase}

The second phase started at about 02:44 UT and lasted after the flare maximum. The emission in energy range of $100-300 \mathrm{keV}$ was observed during this phase. The emission in lower energy range was dominated by source $\mathrm{N}$, which was observed up to $30-35 \mathrm{keV}$. At the flare maximum a second source, S, was observed and brightened with time (Figure 5). Several minutes after the maximum of the flare, the source $S$ dominated emission in energy up to $25 \mathrm{keV}$. For some time we observed only one elongated source and there was no possibility to separate it into sources $\mathrm{N}$ and $\mathrm{S}$. The emission from the footpoints was very strong, especially at the beginning of the second phase (Figure 6). The separation between the footpoints was smaller than during the first phase. At the beginning of the second phase FPN was stronger than FPS and was observed in the energy range up to $200-300 \mathrm{keV}$. FPS was observed in the energy range up to $70-80 \mathrm{keV}$ after the flare maximum (at about 03:07-03:08 UT).

\subsubsection{Physical Parameters}

We calculated the temperature $T_{R}$, emission measure $\mathrm{EM}_{\mathrm{R}}$, and non-thermal energy parameters of both LTSs from imaging spectroscopy (see an example of spectra in Figure 2, right panel). The source $\mathrm{N}$ was observed during the whole rising phase and was brighter than source $\mathrm{S}$ during most of the time. The plasma in the LTS $\mathrm{N}$ was hot at the beginning, $T_{\mathrm{R}}>25 \mathrm{MK}$, and cooler after the flare maximum, $T_{\mathrm{R}} \approx 16 \mathrm{MK}$ (Table 2 ). The heating rate was also very high $\left(E_{\mathrm{H}}>15 \mathrm{erg} \mathrm{cm}^{-3} \mathrm{~s}^{-1}\right.$ at the beginning and $E_{\mathrm{H}}<2.5 \mathrm{erg} \mathrm{cm}^{-3} \mathrm{~s}^{-1}$ after the flare maximum). These values of the heating rate are much higher than the val- 

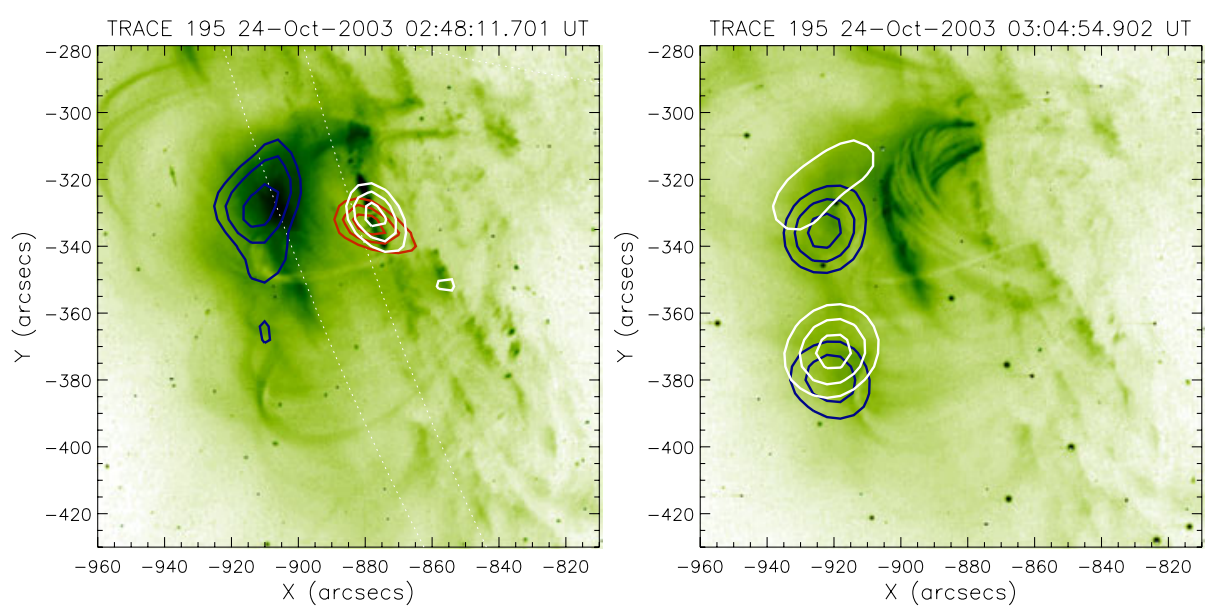

Figure 5 TRACE $195 \AA$ images of the 24 October 2003 flare during the second phase. Contours show the emission in the $10-11 \mathrm{keV}$ range (blue), $20-25 \mathrm{keV}$ range (white) and $100-120 \mathrm{keV}$ range (red) observed with RHESSI. The contours are for $50 \%, 70 \%$ and $90 \%$ of maximum emission.
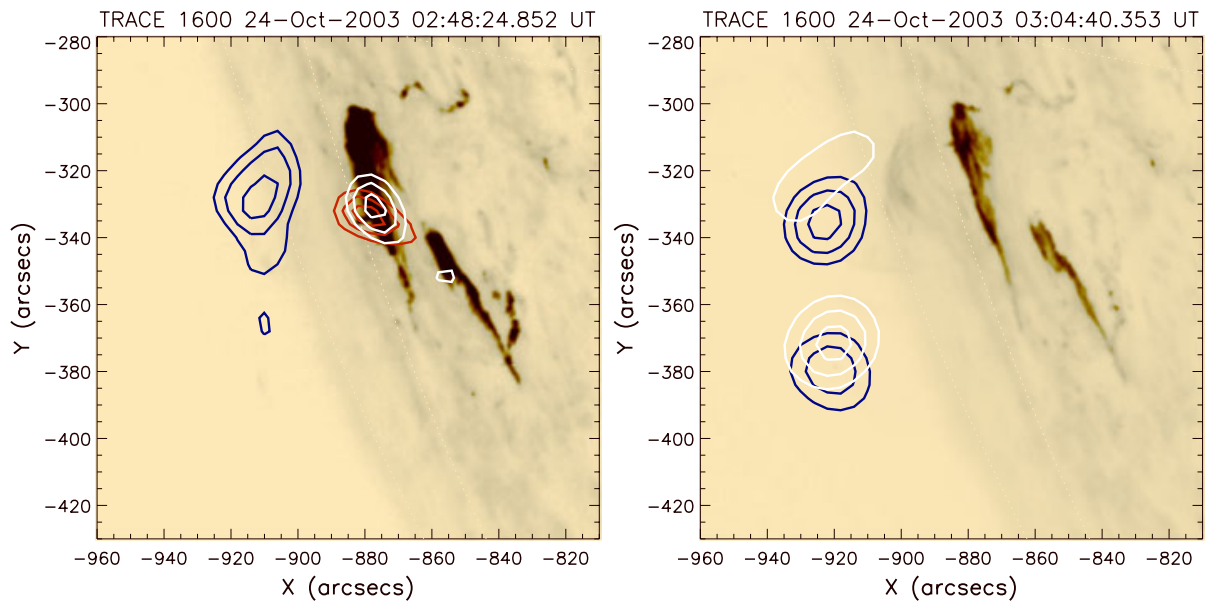

Figure 6 TRACE $1600 \AA$ images of the 24 October 2003 flare during the second phase. Contours show the emission in the 10-11 keV range (blue) and 20-25 keV range (white) and $100-120 \mathrm{keV}$ range (red) observed with RHESSI. The contours are for $50 \%, 70 \%$ and $90 \%$ of maximum emission.

ues obtained from Yohkoh observations, due to limited Yohkoh/SXT sensitivity to higher temperature plasmas. After reaching its maximum value (at the beginning of the flare), the heating rate decreased with a characteristic time $\tau=1240 \mathrm{~s}$. The rate of the non-thermal energy release per unit volume for source $\mathrm{N}$ was about $5 \mathrm{erg} \mathrm{cm}^{-3} \mathrm{~s}^{-1}$ during the rising phase.

Source $\mathrm{S}$ was observed mostly during the second phase. The maximum value of temperature of source $\mathrm{S}$ was $\approx 21 \mathrm{MK}$ and decreased very slowly to $\approx 18 \mathrm{MK}$ after the flare maximum. After the flare maximum, source $\mathrm{S}$ remained hotter and brighter than source $\mathrm{N}$ and became the dominant source. 

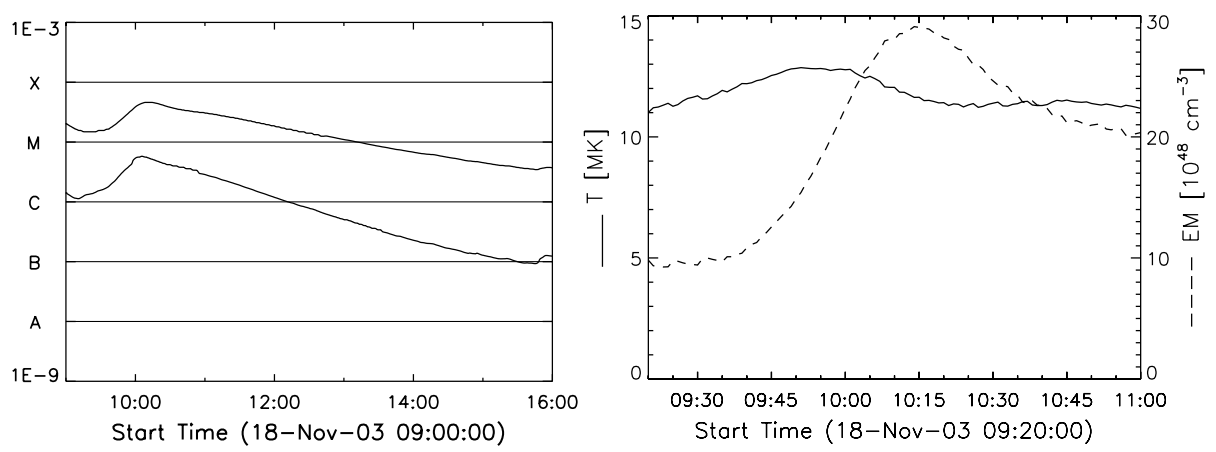

Figure 7 Left: GOES X-ray fluxes (upper curve: $1-8 \AA$, lower curve: $0.5-4 \AA$ ). Right: Temperature and emission measure during the rise phase of the 18 November 2003 flare obtained from the GOES/XRS data.

\subsection{Flare of 18 November 2003}

The flare occurred in active region NOAA 10506 at the eastern solar limb. It began at 09:23 UT. The SXR flux increased slowly and reached its maximum value (GOES class M4.5) at 10:11 UT (Figure 7, left panel). The GOES fluxes were used to calculate the temperature $T_{\mathrm{G}}$, emission measure $\mathrm{EM}_{\mathrm{G}}$, and the time difference between their maxima. The temperature increased slowly and reached its maximum value (12.8 MK) at 09:51 UT (Figure 7, right panel). After that, the temperature slightly dropped (down to $11 \mathrm{MK}$ ). After 10:30 UT, $T_{\mathrm{G}}$ slightly increased which was related to additional emission, seen on the GOES light curve after the maximum. The emission measure reached its maximum value $\left(29 \times 10^{48} \mathrm{~cm}^{-3}\right)$ at 10:14 UT. The time difference between $T_{\mathrm{G}}$ and $\mathrm{EM}_{\mathrm{G}}$ maxima is $23 \mathrm{~min}$.

RHESSI observed the flare almost over the entire rise phase (see Figure 8). Short-lasting pulses were not observed. Only long-lasting emission in the energy range up to $25 \mathrm{keV}$ was observed most of the time. However, for a few moments of time very weak emission in the energy range $25-50 \mathrm{keV}$ was also observed.

In the PIXON images only a loop-top source was observed. This source was spatially correlated with the limb structure seen in the SOHO/EIT images (Figure 9). Only the footpoints of the loops were occulted. The whole structure became visible in the EIT images a few hours after the flare maximum, due to the solar rotation. The structure expanded, and a few hours after the flare maximum, a high arcade of loops was observed. We obtained the location of the HXR source, its size and height above the photosphere from the PIXON images. Emission at a higher energy range $(>20 \mathrm{keV})$ was observed at a higher altitude (Figure 9). Therefore, we calculated the average value of height at each moment of time. The average height increased during the rise phase from $h \approx 20 \mathrm{Mm}$ to $h \approx 50 \mathrm{Mm}$. We calculated the temperature $T_{\mathrm{R}}$, emission measure $\mathrm{EM}_{\mathrm{R}}$, and the parameters for the non-thermal component from the spectra fitting (see Figure 8, right panel). The temperature decreased slowly ( $\approx 19 \mathrm{MK}$ at the beginning, $\approx 17 \mathrm{MK}$ at maximum), which is the characteristic feature of SLDE flares. Physical parameters obtained (see Table 2) allowed us to calculate the rate of thermal energy release $\left(E_{\mathrm{H}} \approx 5 \mathrm{erg} \mathrm{cm}^{-3} \mathrm{~s}^{-1}\right.$ at the beginning, $\approx 1 \mathrm{erg} \mathrm{cm}^{-3} \mathrm{~s}^{-1}$ at the maximum).

During the rise phase a loop-top source was visible up to $40 \mathrm{keV}$ and its emission showed a clear non-thermal component. At about 09:54-09:55 UT the rate of non-thermal energy release per unit volume was about $0.3 \mathrm{erg} \mathrm{cm}^{-3} \mathrm{~s}^{-1}$. 

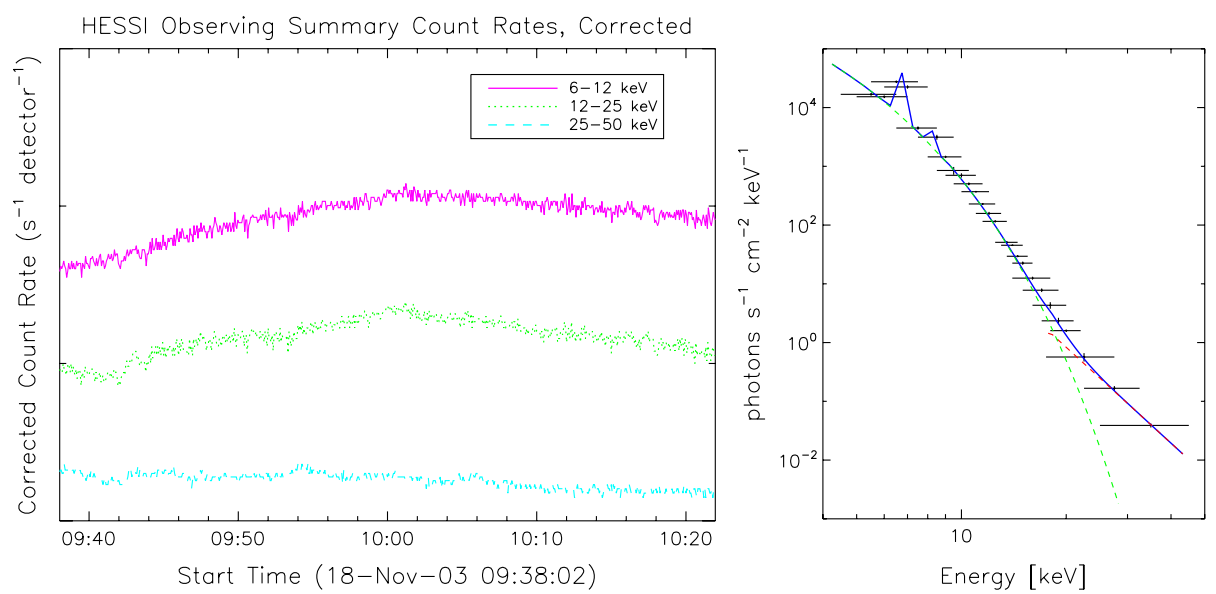

Figure 8 Left: RHESSI light curves of the 18 November 2003 flare. Right: The RHESSI X-ray spectrum of the loop-top source observed during the 18 November 2003 flare at 09:46 UT (horizontal bars correspond to the energy bin widths). This spectrum was fitted using the thermal component (green curve), two spectral line complexes (at $6.7 \mathrm{keV}$ and $8.0 \mathrm{keV}$ ), and a single power-law component (red curve). The sum of all these models, the best-fit model, is represented by the blue curve.
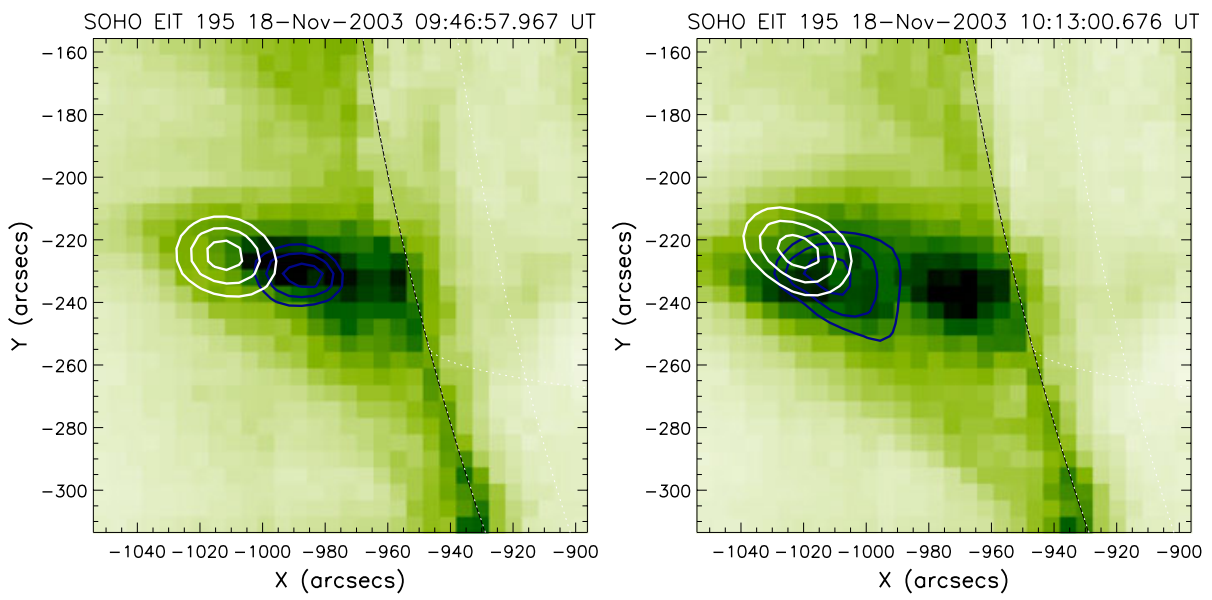

Figure 9 SOHO/EIT $195 \AA$ images of the flare of 18 November 2003 during the rise phase. Contours show the emission in the 10-11 keV range (blue) and 20-25 keV (white) observed with RHESSI. The contours are for $50 \%, 70 \%$ and $90 \%$ of maximum emission.

\subsection{Flare of 6 September 2005}

The flare of 6 September 2005 occurred in an active region NOAA 10808, $\approx 6^{\circ}$ behind the eastern solar limb. The flare (GOES class M1.4) started at 19:32 UT and reached its maximum at 22:02 UT (Figure 10, left panel). The temperature obtained from the GOES/XRS data increased for almost $50 \mathrm{~min}$ and reached maximum (16.2 MK) at 20:23 UT. The decrease in temperature during the rise phase of the flare was very slow (see Figure 10, right panel). The maximum value of emission measure $\left(7.7 \times 10^{48} \mathrm{~cm}^{-3}\right)$ was at 22:02 UT. The 

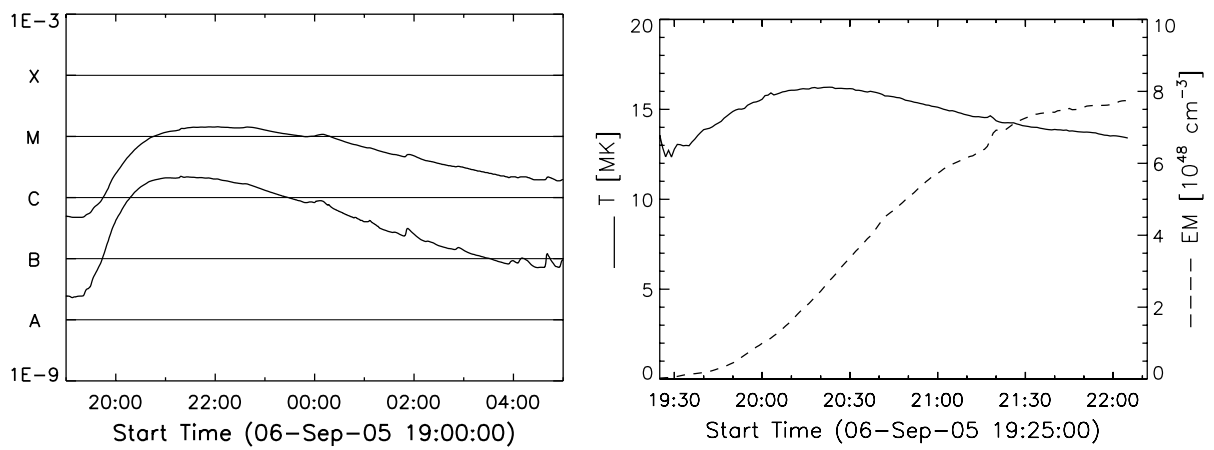

Figure 10 Left: GOES X-ray fluxes (upper curve: $1-8 \AA$, lower curve: $0.5-4 \AA$ ). Right: Temperature and emission measure during the rise phase of the 6 September 2005 flare obtained from the GOES data.

Figure 11 RHESSI light curves for the 6 September 2005 flare.

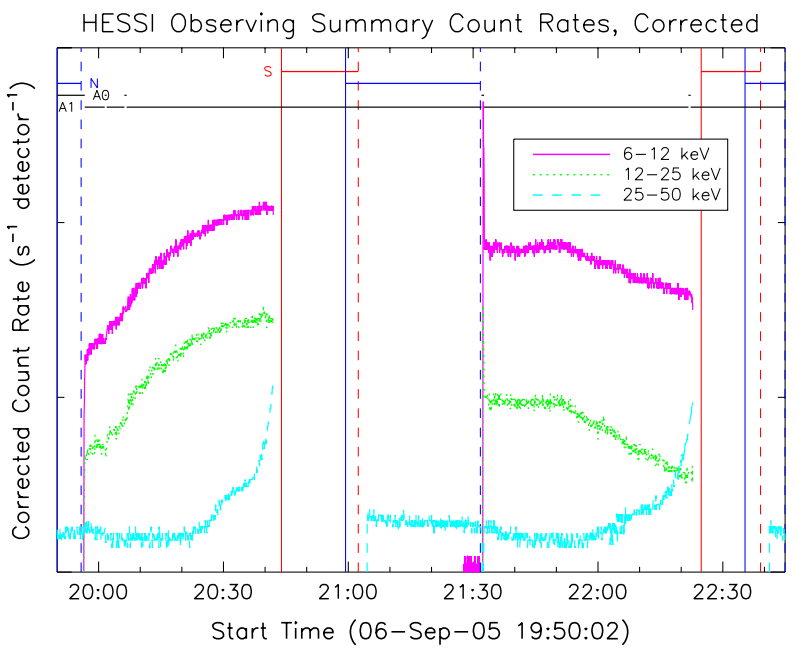

time difference between these two maxima is $101 \mathrm{~min}$, which is the largest $\Delta t$ we have found.

During the rise phase long-lasting HXR emission up to $25 \mathrm{keV}$ was observed (Figure 11) without short-lasting pulses. This flare was slightly occulted, and only the footpoints were behind the limb. The HXR emission source was visible above the loop seen in the SXI image (Figure 12, left panel). TRACE observations ( $3 \mathrm{~h}$ after the flare maximum) show an arcade of loops located near the limb, with loops oriented along the line of sight, which explains the "cusp-like" shape seen in SXI images. From the RHESSI PIXON images we determined the size of the LTS $(\approx 16-20 \mathrm{Mm})$ and its height above the photosphere $(>70 \mathrm{Mm})$. The results are shown in Table 2 . We performed imaging spectroscopy to obtain the physical parameters of the LTS. A spectrum with fitted model (thermal component plus lines at $6 \mathrm{keV}$ and $8 \mathrm{keV}$ ) is shown in Figure 12 , right panel. The temperatures obtained $(\approx 20 \mathrm{MK}$ at the beginning and $\approx 15 \mathrm{MK}$ at the maximum) are higher than those obtained from the GOES/XRS data, which is due to different temperature sensitivities of these two instruments. Slow changes of temperature are a common feature for GOES and RHESSI observations of this flare. The obtained temperature and emission measure (from spectra fitting), size of LTS and height 

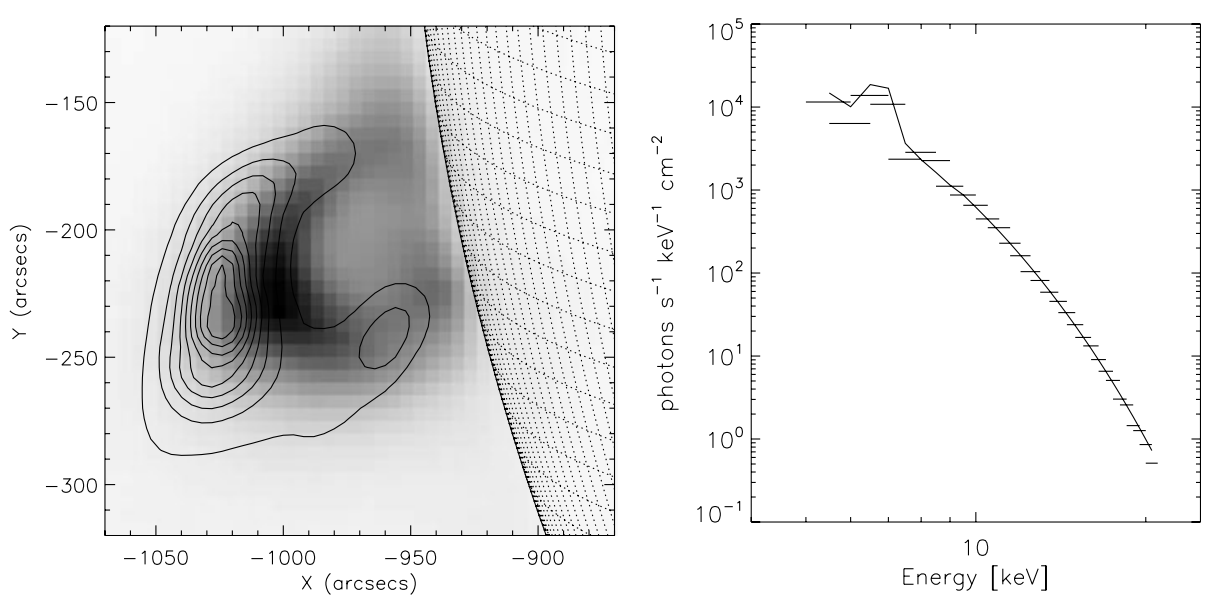

Figure 12 Left: GOES/SXI image of the 6 September 2005 flare during the rise phase. Contours show the emission in the 8-9 keV range observed with RHESSI. The contours are for $10 \%, 20 \%, 30 \%, 40 \%$, $50 \%, 60 \%, 70 \%, 80 \%$ and $90 \%$ of maximum emission. Right: The RHESSI X-ray spectrum of the loop-top source observed during the 6 September 2005 flare at 20:37 UT (horizontal bars correspond to the energy bin widths). This spectrum was fitted using the thermal component and two spectral line complexes (at $6.7 \mathrm{keV}$ and $8.0 \mathrm{keV}$ ). The sum of all these models, the best-fit model, is represented by the black curve.

above the photosphere (from images) allowed us to inspect the energy balance. The value of the heating rate obtained is low (Table 2) and decreases with the characteristic time of $4430 \mathrm{~s}$, which explains the very long rising phase.

\section{Summary}

Slow long-duration events (SLDEs) are flares characterized by long rising phase and smooth HXR emission. We used RHESSI and GOES/XRS data to obtain the physical parameters of the loop-top sources of such flares. TRACE, SOHO/EIT and GOES/SXI images helped us to investigate the morphology of the flares.

Our analysis can be summarized as follows:

- Using RHESSI and GOES/XRS observations, we have confirmed that the characteristic feature of the SLDEs is a large time interval, $\Delta t>20 \mathrm{~min}$, between the maximum of temperature and the maximum of emission measure.

- As other LDEs, slow LDEs often occur in arcades of loops. The height of these structures are high $(h \approx 25-50 \mathrm{Mm})$.

- In almost all cases (except for flare No. 4), long-lasting HXR emission was observed. The flare with the longest rise phase (No. 5) was $\approx 6^{\circ}$ behind the limb and we did not observe their footpoints. In four cases (No. 1, 2, 3, 4), non-thermal emission from the loop-top sources was also observed.

- In flares No. 3 and 4 the rate of non-thermal electron energy release per unit volume (from LTS) was comparable to the heating rate.

- For all analyzed flares we calculated the rate of thermal energy release. The obtained values of $E_{\mathrm{H}}$ are larger than those previous obtained from SXT data (Ba̧k-Stęślicka and Jakimiec, 2005). This difference may well be caused by the fact that SXT had limited 
Table 2 Physical parameters of LTS obtained from the RHESSI data.

\begin{tabular}{|c|c|c|c|c|c|c|c|c|}
\hline & $\begin{array}{l}\text { Date } \\
\text { GOES start } \\
\text { GOES max }\end{array}$ & Time & $\begin{array}{l}h \\
{[\mathrm{Mm}]}\end{array}$ & $\begin{array}{l}r \\
{[\mathrm{Mm}]}\end{array}$ & $\begin{array}{l}T_{\mathrm{R}} \\
{[\mathrm{MK}]}\end{array}$ & $\mathrm{EM}_{\mathrm{R}}^{\mathrm{a}}$ & $E_{\mathrm{H}}^{\mathrm{b}}$ & $\begin{array}{l}\tau \\
{[\mathrm{s}]}\end{array}$ \\
\hline & & \multicolumn{7}{|c|}{ Loop-top source $\mathrm{N}$} \\
\hline & & $02: 27$ & 34 & 11.1 & 25.2 & 0.4 & 9.6 & \\
\hline & & $02: 32$ & 27 & 8.8 & 25.6 & 2.4 & 15.3 & 1240 \\
\hline & & $02: 48$ & 25 & 8.9 & 19.4 & 6.9 & 6.3 & \\
\hline \multirow[t]{5}{*}{1} & 24 Oct. 2003 & 03:04 & 42 & 8.2 & 16.5 & 5.4 & 2.2 & \\
\hline & $02: 19$ & Loop- & ource $S$ & & & & & \\
\hline & $02: 54$ & $02: 54$ & 47 & 4.0 & 20.8 & 1.6 & 9.3 & \\
\hline & & 03:02 & 46 & 7.6 & 21.0 & 1.3 & 5.1 & \\
\hline & & $03: 10$ & 50 & 6.3 & 18.6 & 1.9 & 3.6 & \\
\hline \multirow[t]{4}{*}{2} & 18 Nov. 2003 & 09:40 & 21 & 12.0 & 19.2 & 0.6 & 5.1 & \\
\hline & $09: 23$ & 09:50 & 30 & 11.4 & 17.7 & 2.4 & 2.8 & 2110 \\
\hline & $10: 11$ & 10:01 & 33 & 13.9 & 17.6 & 3.9 & 2.1 & \\
\hline & & $10: 10$ & 40 & 12.2 & 17.4 & 3.9 & 2.0 & \\
\hline \multirow[t]{4}{*}{3} & 13 Jul. 2005 & $14: 16$ & 38 & 8.5 & 23.5 & 2.3 & 7.4 & \\
\hline & $14: 01$ & $14: 23$ & 41 & 9.0 & 22.1 & 4.7 & 5.3 & 2000 \\
\hline & $14: 49$ & $14: 32$ & 39 & 9.3 & 22.0 & 7.1 & 5.3 & \\
\hline & & $14: 39$ & 40 & 9.8 & 20.1 & 10.0 & 3.6 & \\
\hline \multirow[t]{5}{*}{4} & 23 Aug. 2005 & $14: 20$ & 27 & 9.2 & 19.7 & 0.04 & 5.9 & \\
\hline & $14: 19$ & $14: 25$ & 25 & 7.8 & 24.3 & 0.5 & 14.5 & 650 \\
\hline & $14: 44$ & $14: 29$ & 25 & 7.6 & 21.4 & 2.7 & 11.0 & \\
\hline & & $14: 33$ & 28 & 7.4 & 20.6 & 3.8 & 7.8 & \\
\hline & & $19: 58$ & 72 & 19.8 & 20.3 & 0.2 & 1.0 & \\
\hline \multirow[t]{5}{*}{5} & 6 Sep. 2005 & $20: 17$ & 72 & 16.4 & 20.8 & 0.4 & 1.3 & \\
\hline & $19: 32$ & $20: 37$ & 77 & 17.7 & 20.4 & 0.8 & 1.1 & 4430 \\
\hline & $22: 02$ & $21: 34$ & 84 & 19.0 & 16.8 & 1.5 & 0.5 & \\
\hline & & $21: 49$ & 84 & 19.4 & 16.2 & 1.5 & 0.4 & \\
\hline & & $22: 05$ & 82 & 20.3 & 15.2 & 1.9 & 0.3 & \\
\hline \multirow[t]{3}{*}{6} & 25 Jan. 2007 & $06: 47$ & 27 & 12.8 & 15.8 & 0.1 & 1.6 & \\
\hline & $06: 33$ & $06: 55$ & 29 & 12.4 & 13.7 & 1.5 & 1.0 & 1100 \\
\hline & $07: 14$ & 07:00 & 29 & 14.0 & 12.9 & 2.4 & 0.7 & \\
\hline
\end{tabular}

${ }^{\mathrm{a}} \mathrm{EM}$ in $10^{48} \mathrm{~cm}^{-3}$.

${ }^{\mathrm{b}} E_{\mathrm{H}}$ in $\mathrm{erg} \mathrm{cm}^{-3} \mathrm{~s}^{-1}$.

sensitivity to higher temperature plasmas ( $>10 \mathrm{MK}$ ), which usually led to an underestimation of the flare temperature. RHESSI data enable us to estimate much more reliable (higher) values of plasma temperature and therefore higher values of $E_{\mathrm{H}}$.

- We calculated the characteristic time $\tau$ of the decrease in the heating function after reaching its maximum value. The long duration of the rising phase of SLDEs is consistent with a very slow decrease of $E_{\mathrm{H}}$ during that phase. In most cases the characteristic time of the 
decrease in $E_{\mathrm{H}}$ is larger than $1000 \mathrm{~s}$. We obtained the highest value of $\tau$ for the flare with the longest rising phase (No. 5). We obtained the lowest value of $\tau$ for the flare with the shortest rise phase (No. 4).

- Assuming that $E_{\mathrm{H}}$ is equal to the minimum value of $E_{\mathrm{H}}$ calculated for the rising phase, and using the average volume of LTS and the duration of the rising phase obtained from GOES/XRS data, we estimated the total thermal energy released during the rising phase. In our flare samples the total thermal energy is at least $10^{31} \mathrm{erg}$. The same magnitude of energy is released during the decay phase of LDEs (Kołomański, Mrozek, and Ba̧kStęślicka, 2011). This value is larger than the value of the total thermal energy released during the rise phase of short-rising flares (Jiang et al., 2006). Those authors obtained the values not higher than $10^{30} \mathrm{erg}$.

Acknowledgements The RHESSI satellite is NASA Small Explorer (SMEX) mission. We thank Professor Jerzy Jakimiec for many inspiring discussions and also thank Barbara Cader-Sroka for editorial remarks. We thank an anonymous referee for useful comments and suggestions. This investigation has been supported by a Polish Ministry of Science and High Education, grant No. N203 193733.

Open Access This article is distributed under the terms of the Creative Commons Attribution Noncommercial License which permits any noncommercial use, distribution, and reproduction in any medium, provided the original author(s) and source are credited.

\section{References}

Aschwanden, M.J.: 2005, Physics of the Solar Corona: An Introduction, Praxis, Chichester, 561.

Bąk-Stęślicka, U.: 2007, Analysis of the Solar Flares Evolution, Ph.D. thesis, University of Wrocław.

Ba̧k-Stȩślicka, U., Jakimiec, J.: 2005, Solar Phys. 231, 95.

Delaboudinière, J.-P., Artzner, G.E., Brunaud, J., Gabriel, A.H., Hochedez, J.F., Millier, F., et al.: 1995, Solar Phys. 162, 19.

Dere, K.P., Landi, E., Young, P.R., Del Zanna, G., Landini, M., Mason, H.E.: 2009, Astron. Astrophys. 498, 915.

Feldman, U., Seely, J.F., Doschek, G.A., Brown, C.M., Phillips, K.J.H., Lang, J.: 1995, Astrophys. J. 446, 860.

Gallagher, P.T., Dennis, B.R., Krucker, S., Schwartz, R.A., Tolbert, A.K.: 2002, Solar Phys. $210,341$.

Handy, B.N., Acton, L.W., Kankelborg, C.C.: 1999, Solar Phys. 187, 229.

Harra-Murnion, L.K., Schmieder, B., van Driel-Gesztelyi, L.: 1998, Astron. Astrophys. 337, 911.

Hill, S.M., Pizzo, V.J., Balch, C.C., Biesecker, D.A., Bornmann, P., Hildner, E., et al.: 2005, Solar Phys. 226, 255.

Hudson, H.S., McKenzie, D.E.: 2000, In: Ramaty, R., Mandzhavidze, N. (eds.) High Energy Solar Physics Workshop - Anticipating HESSI, ASP Conf. Ser. 206, 221.

Hudson, H.S., McKenzie, D.E.: 2001, Earth Planets Space 53, 581.

Hudson, H.S., Acton, L.W., Freeland, S.L.: 1996, Astrophys. J. 470, 629.

Hudson, H.S., Canfield, R.C., Kane, S.R.: 1978, Solar Phys. 60, 137.

Hurford, G.J., Schmahl, E.J., Schwartz, R.H.: 2002, Solar Phys. $210,61$.

Isobe, H., Yokoyama, T., Shimojo, M., Morimoto, T., Kozu, H., Eto, S., Narukage, N., Shibata, K.: 2002, Astrophys. J. 566, 528.

Jakimiec, J., Tomczak, M., Fludra, A., Falewicz, R.: 1997, Adv. Space Res. 20, 2341.

Jiang, Y.W., Liu, S., Liu, W., Petrosian, V.: 2006, Astrophys. J. 638, 1140.

Joshi, B., Veronig, A., Cho, K.-S., Bong, S.C., Somov, B.V., Moon, Y.-J., Lee, J.-W., Manoharan, P.K., Kim, Y.-H.: 2009, Astrophys. J. 706, 1438.

Kahler, S.: 1977, Astrophys. J. 214, 891.

Kołomański, S.: 2007a, Astron. Astrophys. 465, 1021.

Kołomański, S.: 2007b, Astron. Astrophys. 465, 1035.

Kołomański, S., Mrozek, T., Ba̧k-Stęślicka, U.: 2011, Astron. Astrophys. 531(A57), 1-11.

Kosugi, T., Masuda, S., Makishima, K., Inda, M., Murakami, T., Dotani, T., Ogawara, Y., Sakao, T., Kai, K., Nakajima, H.: 1991, Solar Phys. 136, 17.

Li, H., Li, Y.: 2008, Adv. Space Res. 41, 962. 
Lin, R.P., Dennis, B.R., Hurford, G.J., Smith, D.M., Zehnder, A., Harvey, P.R., et al.: 2002, Solar Phys. 210, 3.

McKenzie, D.E.: 2000, Solar Phys. 195, 38.

McKenzie, D.E., Hudson, H.S.: 1999, Astrophys. J. Lett. 519, L93.

Phillips, K.J.H., Chifor, C., Landi, E.: 2005, Astrophys. J. 626, 1110.

Phillips, K.J.H., Feldman, U., Harra, L.K.: 2005, Astrophys. J. 634, 641.

Puetter, R.C., Yahil, A.: 1999, In: Mehringer, D.M. (ed.) Astronomical Data Analysis Software and Systems VIII, ASP Conf. Ser. 172, 307.

Sheeley, N.R. Jr., Bohlin, J.D., Brueckner, G.E., Purcell, J.D., Scherrer, V.E., Tousey, R., et al.: 1975, Solar Phys. 45, 377.

Tomczak, M.: 1997, Astron. Astrophys. 317, 223.

Tsuneta, S., Acton, L., Bruner, M., Lemen, J., Brown, W., Caravalho, R., et al.: 1991, Solar Phys. $136,37$.

Tsuneta, S., Hara, H., Shimizu, T., Acton, L.W., Strong, K.T., et al.: 1992, Publ. Astron. Soc. Japan 44, 63. 